\title{
Nonequilibrium quantum order at infinite temperature: Spatiotemporal correlations and their generating functions
}

\author{
Sthitadhi Roy ${ }^{1,2, *}$ and Achilleas Lazarides ${ }^{3, \dagger}$ \\ ${ }^{1}$ Physical and Theoretical Chemistry, Oxford University, South Parks Road, Oxford OX1 3QZ, United Kingdom \\ ${ }^{2}$ Rudolf Peierls Centre for Theoretical Physics, Clarendon Laboratory, Oxford University, Parks Road, Oxford OX1 3PU, United Kingdom \\ ${ }^{3}$ Max-Planck-Institut für Physik Komplexer Systeme, Nöthnitzer Straße 38, 01187 Dresden, Germany
}

(Received 16 May 2018; published 28 August 2018)

\begin{abstract}
Localization-protected quantum order extends the idea of symmetry breaking and order in ground states to individual eigenstates at arbitrary energy. Examples include many-body localized static and $\pi$ spin glasses in Floquet systems. Such order is inherently dynamical and difficult to detect as the order parameter typically varies randomly between different eigenstates, requiring specific superpositions of eigenstates to be targeted by the initial state. We show that two-time correlators overcome this, reflecting the presence or absence of eigenstate order even in fully mixed, infinite temperature states. We show how spatiotemporal correlators are generated by the recently introduced dynamical potentials, demonstrating this explicitly using an Ising spin glass and a Floquet $\pi$ spin glass and focusing on features mirroring those of equilibrium statistical mechanics such as bimodal potentials in the symmetry-broken phase.
\end{abstract}

DOI: 10.1103/PhysRevB.98.064208

\section{INTRODUCTION}

Traditionally, phases of matter and transitions between them have been a notion restricted to equilibrium and understood largely using Landau's theory of broken symmetries [1]. Very recently, many-body localization [2-13] has led to the introduction of localization-protected quantum order [14] which has ushered in a new paradigm of order in quantum matter (see Sec. II A 1 for a definition). In phases hosting such order in static systems, individual many-body eigenstates at arbitrary energy densities spontaneously break symmetries of the Hamiltonian and usually exhibit random glassy order [14-17]. The notion of eigenstate phases has also been extended to the class of periodically driven, or Floquet, systems, where fundamentally new phases have been proposed [18-20] and observed [21-24].

Such novel out-of-equilibrium phases raise some fundamental questions, two of which we address in this work.

(i) The presence of order in all eigenstates of the system naturally suggests that dynamical order parameters be used to characterize the phases. However, the eigenstate order is random over both space and energy, and hence in generic initial states having overlap with many eigenstates, the order gets washed out in time-dependent expectations of observables. Therefore probes robust to initial conditions, in particular extreme situations like infinite temperature ensembles, are of interest.

(ii) As eigenstate phases and transitions are dynamic, rather than thermodynamic, phenomena, it is not possible to study them with the usual tools of statistical mechanics and thermodynamics such as effective potentials and free energies.

\footnotetext{
*sthitadhi.roy@chem.ox.ac.uk

†acl@pks.mpg.de
}

Hence a framework for studying the statistical mechanics of such dynamical order is naturally an interesting question.

We resolve the first of the two issues by showing that out-ofequilibrium spatiotemporal correlations robustly encode the presence or the absence of eigenstate order, remaining a good diagnostic even with the system in an infinite-temperature state where its density matrix is proportional to identity. Hence, the presence of an eigenstate-ordered phase can be diagnosed for arbitrary initial states. This is of practical importance in cases where coupling to an external environment is significant (such as in solid-state systems, trapped-ion systems, etc.) since the resulting thermal state will necessarily involve an incoherent mixture of eigenstates.

The usefulness of spatiotemporal correlations hints towards a possible direction for addressing the second question. In order to develop a statistical mechanics-like framework for eigenstate phases, one should try to construct effective potentials which act as generating functions for such correlations [25], much like the free energy in equilibrium. We do this using the framework of dynamical potentials. Drawing inspiration from the so-called $s$ ensemble [26,27], the framework was introduced in a previous work [28] for nonequilibrium quantum many-body phases, but only in the context of pure states. Here we generalize the framework to mixed states such as the infinite temperature states already mentioned. The connection to spatiotemporal correlations lies in the fact that these potentials can be recast as probability distributions, moments of which correspond to various spatiotemporal correlations. These potentials and hence the probability distributions are found to exhibit qualitatively different behaviors in different eigenstate ordered phases. For example, in a $\mathbb{Z}_{2}$-symmetric Ising spin glass, which spontaneously breaks the $\mathbb{Z}_{2}$ symmetry in the spin-glass phase at all energy densities, we find that appropriately constructed distributions are bimodal, whereas in the paramagnet phase the same distributions are unimodal with 
a vanishing width in the thermodynamic limit. The bimodal distribution guarantees a finite two-point (in space and time) correlation function for finite systems and hence is a signature for spontaneous symmetry breaking in the thermodynamic limit $[29,30]$. The unimodal distribution with a vanishing width in the paramagnet phase on the other hand shows the absence of any long-ranged (in space and time) correlation or order. This is analogous to double (single)-well free-energy potentials in ordered (disordered) phases in equilibrium statistical mechanics. The framework then provides a statistical mechanics-esque way of describing eigenstate order phases macroscopically which is also robust to infinite temperature ensembles and hence is expected to work for any generic initial condition and constitutes the central result of this work.

To concretely demonstrate our results, we explicitly construct the potentials for the two prototypical examples of eigenstate ordered systems, namely, a $\mathbb{Z}_{2}$-symmetric disordered Ising chain hosting a spin glass-paramagnet phase transition [14] and its periodically driven cousin hosting a $\pi$ spin glass/discrete time crystal phase [18] exclusive to Floquet systems.

The rest of the paper is organized as follows. In Sec. II, we discuss the phenomenology of eigenstate order in a disordered Ising spin chain and its periodically driven version, and we demonstrate how spatiotemporal correlations at infinite temperature encode the eigenstate order. Section III generalizes the framework of dynamical potentials for mixed states, and their explicit numerical constructions and discussions on the results for the two models are presented in Sec. IV. In Sec. V, we display results for a particular operator which leads to bimodal potentials (and probability distributions) in the eigenstate ordered phases. Finally we conclude with a summary and outlook in Sec. VI.

\section{SPATIOTEMPORAL CORRELATIONS AND QUANTUM ORDER}

\section{A. Phenomenology of eigenstate order}

\section{Static}

The paradigmatic system displaying localization protected quantum order is the $\mathbb{Z}_{2}$-symmetric disordered Ising chain in one dimension with the Hamiltonian [14]

$$
\mathcal{H}_{\mathrm{ISG}}=\sum_{\ell}\left[J_{\ell} \sigma_{\ell}^{z} \sigma_{\ell+1}^{z}+J_{x} \sigma_{\ell}^{x} \sigma_{\ell+1}^{x}+h_{\ell} \sigma_{\ell}^{x}\right],
$$

where $J_{\ell} \in\left[J_{z}-J, J_{z}+J\right]$ and $h_{\ell} \in\left[h_{x}-h, h_{x}+h\right]$ denote random spin-spin interactions and fields, respectively. For $J \gg J_{z}, J_{x}, h$, the model hosts an eigenstate ordered phase with Ising spin glass order which can be captured by an Edwards-Anderson order parameter. In this phase, disorder pins the domain walls spatially, leading to random glassy order in the system. As a result, the $\mathbb{Z}_{2}$ symmetry is spontaneously broken at all energy densities (rather than just in the ground states, as in the clean Ising ferromagnet). This type of order is called eigenstate order [14].

Concretely, since the parity operator $\mathcal{P}=\prod_{\ell} \sigma_{\ell}^{x}$ commutes with the Hamiltonian (1), the eigenstates of $\mathcal{H}_{\text {ISG }}$ are eigenstates of $\mathcal{P}$ simultaneously. In the spin glass phase the eigenstates of $\mathcal{H}_{\text {ISG }}$ are each twofold degenerate (up to corrections exponentially small in system size) with each member of the pair having opposite parity. We write these states as $|\varepsilon, \pm\rangle$, where

$$
\mathcal{H}_{\mathrm{ISG}}|\varepsilon, \pm\rangle=\varepsilon|\varepsilon, \pm\rangle ; \mathcal{P}|\varepsilon, \pm\rangle= \pm|\varepsilon, \pm\rangle .
$$

Each eigenstate has long-ranged order along the $\sigma^{z}$ direction:

$$
\left\langle\varepsilon \pm\left|\sigma_{i}^{z} \sigma_{j}^{z}\right| \varepsilon, \pm\right\rangle \neq 0 ;|i-j| \rightarrow \infty .
$$

The presence of spontaneous symmetry-broken order in the thermodynamic limit becomes explicit if the eigenstates $|\varepsilon, \pm\rangle$ are expressed in the symmetry-broken basis

$$
|\varepsilon, \pm\rangle=\frac{1}{\sqrt{2}}[|\varepsilon, \uparrow\rangle \pm|\varepsilon, \downarrow\rangle], \quad|\varepsilon, \uparrow\rangle=\mathcal{P}|\varepsilon, \downarrow\rangle,
$$

where the order is evident:

$$
\left\langle\varepsilon, \uparrow\left|\sigma_{i}^{z}\right| \varepsilon, \uparrow\right\rangle=-\left\langle\varepsilon, \downarrow\left|\sigma_{i}^{z}\right| \varepsilon, \downarrow\right\rangle=\eta_{i, \varepsilon} \neq 0 .
$$

The $\eta_{i, \varepsilon}$ are not smooth functions of $\varepsilon$, changing randomly between eigenstates close in energy. They also remain finite with increasing system size. Equations (4) and (5) imply

$$
\left\langle\varepsilon, \pm\left|\sigma_{i}^{z}\right| \varepsilon, \mp\right\rangle=\eta_{i, \varepsilon} .
$$

The model also hosts a paramagnetic phase for $h, h_{x} \gg$ $J, J_{x}$, where the $\eta_{i, \varepsilon}$ 's defined in Eq. (5) vanish in the thermodynamic limit.

\section{Floquet}

The presence of $\mathbb{Z}_{2}$ symmetry and localization also allows for a fundamentally different out-of-equilibrium phase, called the $\pi$ spin glass or the "discrete time crystal," in the periodically driven cousin of the model. Note that disorder here is fundamentally important to prevent heating under the driving [31-37]. The discrete time crystalline behavior of the system shows up in the form of a subharmonic signal. The model is described by the time-dependent Hamiltonian of the unit period:

$$
\mathcal{H}_{\pi \mathrm{SG}}(t)=\left\{\begin{array}{l}
\sum_{\ell}\left[J_{\ell} \sigma_{\ell}^{z} \sigma_{\ell+1}^{z}+J_{x} \sigma_{\ell}^{x} \sigma_{\ell+1}^{x}\right] ; n \leqslant t<n+\frac{1}{2}, \\
\sum_{\ell}\left[h_{\ell} \sigma_{\ell}^{x}+J_{x} \sigma_{\ell}^{x} \sigma_{\ell+1}^{x}\right] ; n+\frac{1}{2} \leqslant t<n+1,
\end{array}\right.
$$

where $n$ takes integer values. Much of the phenomenology of the spatial glassy order of the Ising spin glass carries over to the eigenstates of the Floquet unitary operator $U_{F}=$ $e^{-i \int_{0}^{1} d t \mathcal{H}_{\pi \mathrm{SG}}(t)}$, which again come in pairs:

$$
U_{F}|\varepsilon, \pm\rangle= \pm e^{-i \varepsilon}|\varepsilon, \pm\rangle,
$$

where the quantity $\varepsilon$ plays the role of energy, is only defined modulo the driving frequency $2 \pi$, and is therefore called the quasienergy [38]. The difference from the static case is that the two parity-related eigenstates $|\varepsilon, \pm\rangle$ are no longer degenerate in quasienergy but rather are separated by half the driving frequency, that is, by $\pi$. Switching again to the symmetry-broken basis, the extra $\pi$ phase between the two eigenstates leads to the stroboscopic evolution

$$
U_{F}^{n}|\varepsilon, \uparrow / \downarrow\rangle=e^{-i n \varepsilon}|\varepsilon, \downarrow / \uparrow\rangle,
$$


and hence $\left\langle\varepsilon, \uparrow / \downarrow\left|\sigma_{i}^{z}(n)\right| \varepsilon, \uparrow / \downarrow\right\rangle= \pm(-1)^{n} \eta_{i, \varepsilon}$ such that the stroboscopic response has a period twice that of the Hamiltonian (7), reducing the discrete time translation symmetry of the Hamiltonian by a factor of 2 . This is a direct consequence of the pairing in the spectrum of $U_{F}$.

In conclusion, the $\pi$ spin glass displays temporal order with a frequency which is half the frequency of driving in addition to the spatial order.

\section{B. Spatiotemporal correlations at infinite temperature}

The eigenstate order in both 0 and $\pi$ spin glass is random, both spatially and between eigenstates. While realistic schemes for preparing the system such that the order is visible (essentially, preparing it in a superposition dominated by two eigenstates forming one of the pairs) are possible, an obvious question is whether there is some signature of this order in high-temperature mixed states. Focusing on the extreme limit of the infinite-temperature state where the density matrix is proportional to identity, we show that the answer to this is affirmative, provided that the appropriate operators defining the potential (the $\mathcal{O}$ of Sec. III) are selected.

Let us begin by showing what the difficulty is and how it is circumvented in the 0 spin glass. The order, indicated by finite $\eta_{i, \varepsilon}$ in Eq. (5), is random in magnitude and sign over space and eigenstates.

As a result, dynamical expectation values of the macroscopic version of the observable, $M_{z}=\sum_{\ell} \sigma_{l}^{z}$, average out to zero: $\left\langle M_{z}(t)\right\rangle_{\infty} \rightarrow 0$ as $t \rightarrow \infty$, where the $\infty$ in the subscript denotes the expectation value over an infinite temperature state, $\left\langle M_{z}(t)\right\rangle_{\infty}=\operatorname{Tr}\left[M_{z}(t) \rho_{\infty}\right]$, with $\rho_{\infty}=\mathbb{I}_{\operatorname{dim}[\mathcal{H}]} / \operatorname{dim}[\mathcal{H}]$. Nevertheless, as we now show, two-time correlations remain finite even at infinite temperatures capturing the presence of spatiotemporal order. This applies to both the static Ising spin glass and the $\pi$ spin glass: Consider the two-time correlator of the longitudinal magnetization

$$
\mathcal{C}_{M_{z}}\left(t_{1}, t_{2}\right)=\operatorname{Tr}\left[M_{z}\left(t_{1}\right) M_{z}\left(t_{2}\right) \rho_{\infty}\right],
$$

which for the Ising spin glass using Eq. (2) can be expressed as

$$
\begin{aligned}
\mathcal{C}_{M_{z}}\left(t_{1}, t_{2}\right)= & \frac{1}{\operatorname{dim}[\mathcal{H}]} \sum_{\varepsilon, \varepsilon^{\prime}, \varepsilon^{\prime \prime} \bullet, \mathbf{\Lambda}, \mathbf{\nabla}= \pm}\left[\left\langle\varepsilon, \bullet\left|M_{z}\right| \varepsilon^{\prime}, \mathbf{\Lambda}\right\rangle\left\langle\varepsilon^{\prime}, \mathbf{\Lambda} \mid \varepsilon^{\prime \prime}, \mathbf{\nabla}\right\rangle\right. \\
& \left.\times\left\langle\varepsilon^{\prime \prime}, \mathbf{\nabla}\left|M_{z}\right| \varepsilon, \bullet\right\rangle e^{i t_{1}\left(\varepsilon-\varepsilon^{\prime}\right)} e^{i t_{2}\left(\varepsilon^{\prime \prime}-\varepsilon\right)}\right] .
\end{aligned}
$$

The time-averaged two-time correlator is then defined as

$$
\overline{\mathcal{C}}_{M_{z}}(t)=\frac{1}{t^{2}} \int_{0}^{t} d t_{1} \int_{0}^{t} d t_{2} \mathcal{C}_{M_{z}}\left(t_{1}, t_{2}\right),
$$

which in the limit of $t \rightarrow \infty$ can be expressed using Eq. (6) as

$$
\begin{aligned}
\overline{\mathcal{C}}_{M_{z}}(t \rightarrow \infty) & =\frac{2}{\operatorname{dim}[\mathcal{H}]} \sum_{\varepsilon} \sum_{i, j=1}^{L} \eta_{i, \varepsilon} \eta_{j, \varepsilon} \\
& \approx \frac{2}{\operatorname{dim}[\mathcal{H}]} \sum_{\varepsilon} \sum_{i}^{L} \eta_{i, \varepsilon}^{2} \sim O(L),
\end{aligned}
$$

where terms of the form $\sum_{i \neq j} \eta_{i, \varepsilon} \eta_{j, \varepsilon}$ vanish in the thermodynamic limit as $\eta_{i, \varepsilon}$ can take random signs. On the other hand, since the average magnitudes of $\eta_{i, \varepsilon}$ do not vanish in the spin glass phase in the thermodynamic limit, terms of the form in Eq. (13) survive and yield an $O(L)$ contribution to the two-time correlator. Contrary to the spin glass phase, in the paramagnetic phase $\eta_{i, \varepsilon}$ vanishes in magnitude in the thermodynamic limit and consequently so does the two-time correlator. Hence, the question of whether the dynamical order in the spin glass phase survives infinite temperature is answered in the affirmative and the two-time correlation of the macroscopic longitudinal magnetization carries the information of the order.

A similar analysis shows the presence of the temporal order in the case of the $\pi$ spin glass, where the stroboscopic two-time correlator can be expressed as

$$
\begin{aligned}
\mathcal{C}_{M_{z}}\left(n_{1}, n_{2}\right)= & \frac{1}{\operatorname{dim}[\mathcal{H}]} \sum_{\varepsilon, \varepsilon^{\prime}, \varepsilon^{\prime \prime}} \sum_{\mathbf{\Lambda}, \mathbf{\nabla}= \pm} e^{i \varepsilon\left(n_{1}-n_{2}\right)}\left[\left\langle\varepsilon,+\left|M_{z}\right| \varepsilon^{\prime}, \mathbf{\Delta}\right\rangle\right. \\
& \times\left\langle\varepsilon^{\prime}, \mathbf{\Delta}\left|U_{F}^{n_{1}-n_{2}}\right| \varepsilon^{\prime \prime}, \mathbf{\nabla}\right\rangle\left\langle\varepsilon^{\prime \prime}, \mathbf{\nabla}\left|M_{z}\right| \varepsilon,+\right\rangle \\
& +\left\langle\varepsilon,-\left|M_{z}\right| \varepsilon^{\prime}, \mathbf{\Delta}\right\rangle\left\langle\varepsilon^{\prime}, \mathbf{\Delta}\left|U_{F}^{n_{1}-n_{2}}\right| \varepsilon^{\prime \prime}, \mathbf{\nabla}\right\rangle \\
& \left.\times\left\langle\varepsilon^{\prime \prime}, \mathbf{\nabla}\left|M_{z}\right| \varepsilon,-\right\rangle(-1)^{n_{1}+n_{2}}\right] .
\end{aligned}
$$

Using Eqs. (6) and (8) in the long-time limit this can be expressed as

$$
\mathcal{C}_{M_{z}}\left(n_{1}, n_{2}\right) \approx(-1)^{n_{1}+n_{2}} \frac{2}{\operatorname{dim}[\mathcal{H}]} \sum_{\varepsilon} \sum_{i}^{L} \eta_{i, \varepsilon}^{2},
$$

which again is $O(L)$ and hence nonvanishing in the thermodynamic limit but, more crucially, oscillates with a period twice the stroboscopic time and hence reflects the discrete time crystalline order in addition to the spatial spin glass order.

\section{DYNAMICAL POTENTIALS FOR MIXED STATES}

Having established that temporal correlation functions are the key towards exposing the eigenstate order at infinite temperature, we generalize the framework of dynamical potentials introduced in Ref. [28] for pure states to mixed states.

Consider a system governed by a Hamiltonian $\mathcal{H}(t)$ and let the observable of interest be $\mathcal{O}$. If the initial state of the system at $t=0$ is described by the density matrix $\rho$, then one can define a functional

$$
\mathcal{Z}_{t}[s]=\operatorname{Tr}\left[\mathcal{T} e^{-i \int_{0}^{t} d t^{\prime} \mathcal{H}^{(s)}\left(t^{\prime}\right)} \rho \tilde{\mathcal{T}} e^{i \int_{0}^{t} d t^{\prime} \mathcal{H}^{(s) \dagger}\left(t^{\prime}\right)}\right],
$$

where

$$
\mathcal{H}^{(s)}(t)=\mathcal{H}(t)-i s(t) \mathcal{O} / 2
$$

and $\mathcal{T}(\tilde{\mathcal{T}})$ denotes (anti-)time orderings. $\mathcal{Z}_{t}[s]$ acts as the moment-generating functional for the correlations of $\mathcal{O}$ as

$$
\begin{gathered}
\left.\frac{\delta \mathcal{Z}}{\delta s(t)}\right|_{s=0}=\operatorname{Tr}[\mathcal{O}(t) \rho], \\
\left.\frac{\delta^{2} \mathcal{Z}}{\delta s\left(t_{1}\right) \delta s\left(t_{2}\right)}\right|_{s=0}=\frac{1}{2}\left\{\operatorname{Tr}\left[\mathcal{O}\left(t_{1}\right) \mathcal{O}\left(t_{2}\right) \rho\right]+t_{1} \leftrightarrow t_{2}\right\},
\end{gathered}
$$


and so on. In the case of a constant $s, \mathcal{Z}(s, t)$ takes on the integrated form of the temporal correlations as

$$
\begin{gathered}
\left.\frac{\partial \mathcal{Z}(s, t)}{\partial s}\right|_{s=0}=\int_{0}^{t} d t^{\prime} \operatorname{Tr}\left[\mathcal{O}\left(t^{\prime}\right) \rho\right], \\
\left.\frac{\partial^{2} \mathcal{Z}(s, t)}{\partial s^{2}}\right|_{s=0}=\int_{0}^{t} d t_{1} \int_{0}^{t} d t_{2} \operatorname{Tr}\left[\mathcal{O}\left(t_{1}\right) \mathcal{O}\left(t_{2}\right) \rho\right],
\end{gathered}
$$

and so on. One can define $\Theta(s, t)=L \theta(s, t)$ as the corresponding cumulant-generating function via

$$
\mathcal{Z}(s, t)=e^{-\Theta(s, t)} .
$$

As in Ref. [28], the moment-generating function can be used to calculate the probability distribution whose moments encode the temporal correlations of various orders. Expressing $\mathcal{Z}(s, t)$ as

$$
\mathcal{Z}(s, t)=\int d m e^{-s m L} P(m, t, L),
$$

where $P(m, t, L) \sim e^{-\Phi(m, t, L)}$, with $\Phi(m, t, L)=L \phi(m, t, L)$, one can simply calculate $P$ from $\phi$ via a Legendre transform:

$$
\phi(m, t)=-\max _{s}[s m-\theta(s, t)] .
$$

The potentials $\theta$ and $\phi$ effectively contain all the information of the dynamics of the system at infinite temperature in the form of temporal correlations. As shown in the following sections, they exhibit qualitatively different behaviors in different phases and hence provide for a macroscopic characterization of such eigenstate phases.

\section{NUMERICAL RESULTS}

As spatiotemporal correlations can show eigenstate order at infinite temperature and the dynamical potentials provide a general framework for studying them, we present pertinent numerical results for the Ising spin glass (1) as well as for the periodically driven model (7). We choose $\mathcal{O}=M_{z}=\sum_{\ell} \sigma_{l}^{z}$, the total longitudinal magnetization.

\section{A. Ising spin glass}

We numerically compute the potentials corresponding to the Ising spin glass for two different parameter values expected to be in the spin glass and paramagnetic phases. The potentials are calculated with $s$ constant in time so we can get time-integrated temporal correlation functions of the form in Eq. (12). The results for the disorder-averaged potential $\Theta_{M_{z}}$ are shown in Figs. 1(a) and 1(b) and they clearly show different behaviors in the two phases. In the spin glass phase, the curvature at $s=0$ increases with increasing $L$, whereas in the paramagnet phase the curvature decreases with increasing $L$. This is shown more clearly in Figs. 1(c) and 1(d) where we plot the second derivative of $\Theta_{M_{z}}$ with respect to $s$ at $s=0$ scaled with $L$ and $t$. The collapse of the data and linear behavior of $\left.\partial_{s}^{2} \Theta\right|_{s=0} / L t$ with $t$ in the spin glass phase suggests a scaling of the form $\left.\partial_{s}^{2} \Theta_{M_{z}}\right|_{s=0} \sim L t^{2}$, reflecting the presence of spin glass order and in agreement with the scaling predicted from the phenomenology in Eq. (13). On the other hand, in the paramagnet phase, not just the second derivative but all derivatives of $\Theta$ with respect to $s$ at $s=0$ seem to
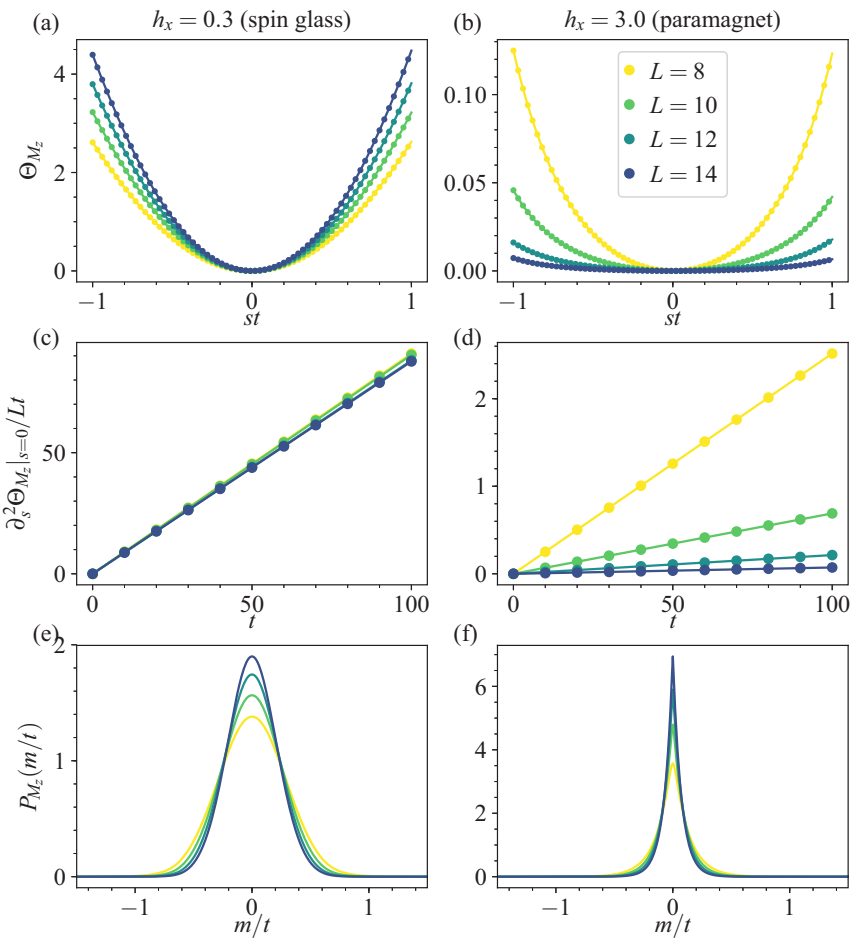

FIG. 1. The dynamical potential $\Theta_{M_{z}}$ as a function of $s$ and $t$ for different system sizes, $L$, in the spin glass (a) and paramagnet (b) phases, respectively. Note that they have opposite trends with increasing $L$ with regard to their curvature at $s=0$. The second derivative of $\Theta_{M_{z}}$ with respect to $s$ at $s=0$ as a function of $t$ scaled with $L$ and $t$ suggests that $\Theta_{M_{z}} \sim s^{2} t^{2} L$ in the spin glass phase (c) and vanishes in the thermodynamic limit in the paramagnet phase (d). The distribution $P_{M_{z}}(m / t)$ shows a Gaussian and exponential behavior in the spin glass phase (e) and the paramagnet phase (f), respectively. Other parameters are $J_{z}=0, J=5, J_{x}=0.3$, and $h=0.3$.

vanish in the thermodynamic limit [see Figs. 1(b) and 1(d)]. Hence, the dynamical potential $\Theta_{M_{z}}$ shows that the eigenstate order can be probed via a macroscopic observable at infinite temperature.

As mentioned in Sec. III, we can also construct the distribution $P$, moments of which yield temporal correlations of all orders. In Figs. 1(e) and 1(f) we show $P$ in both spin glass and paramagnetic phases using Eq. (24). They exhibit qualitatively different behavior: while in the spin glass phase, $P$ has a Gaussian form with standard deviation proportional to $1 / \sqrt{L}$, and in the paramagnetic phase the distribution has an exponential form. The origin of the Gaussian in the spin glass phase can be understood easily because the leading contribution to $\Theta_{M_{z}}$ in the thermodynamic limit is $\sim s^{2} t^{2} L$ and hence the leading contribution to $\Phi$ is $\sim m^{2} L / t^{2}$. On the other hand, in the paramagnet phase, since all derivatives of $\Theta$ with respect to $s$ at $s=0$ decrease with increasing $L$, the distribution decays exponentially away from the mean.

Note that the first derivative of $\Theta$ with respect to $s$ at $s=0$ vanishes in both the phases, indicating that the expectation value of $M_{z}$ as a function of $t$ cannot capture the difference between the spin glass and the paramagnet phase, thus highlighting the importance of multiple-time correlations. This is 

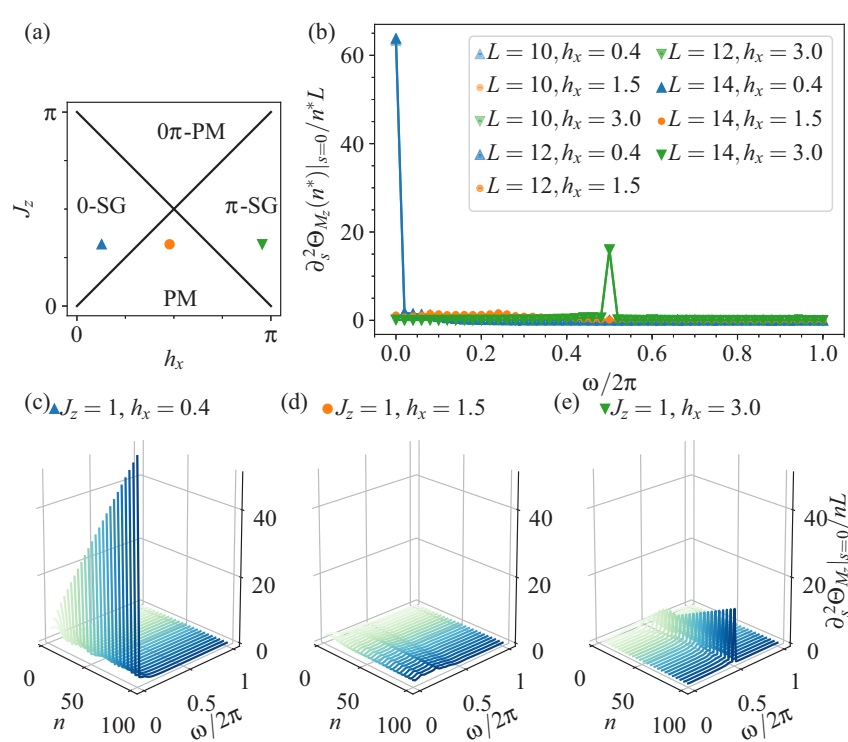

(d) $\bullet J_{z}=1, h_{x}=1.5$

(e) $\stackrel{\omega / 2 \pi}{\nabla} J_{z}=1, h_{x}=3.0$
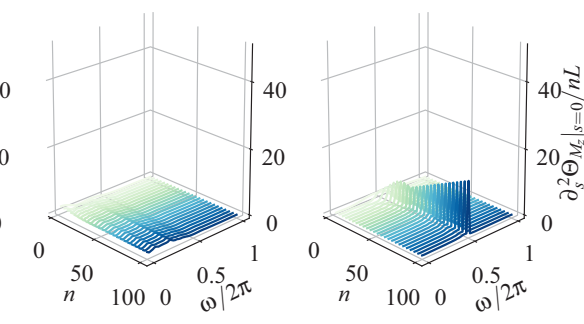

FIG. 2. (a) The phase diagram of the model in Eq. (7) for $J_{x}=0$. The markers show the parameter values of $J_{z}$ and $h_{x}$, corresponding to which the numerical results are shown in the rest of the panels. (b) $\left.\partial_{s}^{2} \Theta_{M_{z}}\left(n^{*}\right)\right|_{s=0} / n^{*} L$ as a function of the frequency $\omega$ at fixed time $n^{*}=100$ for the three different $\left(J_{z}, h_{x}\right)$ values marked in panel (a) for different $L$. The collapse of the data for different $L$ suggests that the response survives in the thermodynamic limit. (c)-(e) The behavior of $\left.\partial_{s}^{2} \Theta_{M_{z}}\right|_{s=0} / n L$ as a function of $\omega$ and $n$ together shows that the integrated two-time correlator grows quadratically with time, if probed at $\omega=0$ and $\pi$ in the 0 and $\pi$ spin glass phases, respectively. Other parameters are $J=5, J_{x}=0.1, h=0.3$, and $L=14$ for panels (c)-(e).

also manifested in the fact that the mean of the distribution $P$ is zero in both the phases.

\section{B. Periodically driven Ising spin glass}

The periodically driven cousin of the disordered Ising model (7) in the limit of $J_{x}=0$ has an exactly known phase diagram shown in Fig. 2(a) and the phases are known to be stable to the presence of interactions provided the system stays in the Floquet many-body localized phase. Since the model is driven periodically in time (with period 1 ) and thus can have nontrivial temporal behavior, we use a time-dependent $s$ of the form

$$
s(t)=s \cos (\omega t),
$$

and hence the potentials now have an additional parameter, namely the frequency, $\omega$, of the source field. With this form of $s(t)$, we calculate the $\Theta_{M_{z}}$ for three parameter values, corresponding to the 0 spin glass (SG) phase, the paramagnet phase, and the $\pi$ spin glass phase, as shown by the markers in Fig. 2(a).

Since the 0 spin glass phase has a phenomenology identical to the Ising spin glass phase discussed in Sec. IV A, $\left.\partial_{s}^{2} \Theta\right|_{s=0} \sim$ $s^{2} n^{2} L$ ( $n$ being the stroboscopic time) for $\omega \rightarrow 0$. This can be inferred from the data for $h_{x}=0.4$ from Figs. 2(b) and 2(c).

More interestingly, in the $\pi$ spin glass phase, a similar response is present for $\omega=\pi$, i.e., at half the frequency of the periodic drive, and hence shows a time crystalline response [Figs. 2(b) and 2(e)]. The response at $\omega=\pi$ can be understood quite simply from Eq. (15) as the two-time correlator $\mathcal{C}_{M_{z}}\left(n_{1}, n_{2}\right)$ has a subharmonic response in both $n_{1}$ and $n_{2}$. However, the more remarkable aspect is that the information of the subharmonic response in the two-time correlator survives even in an infinite temperature ensemble despite the spatial order being random over space and eigenstates.

Finally, in the paramagnet phase, since there is no spatial order anyway, there is no response at any frequency as can be seen in Figs. 2(b) and 2(d).

\section{BIMODALITY AND NONANALYTIC POTENTIALS}

In Sec. III, we saw that the probability distribution for the chosen operator (analogous to the exponential of a thermodynamic potential) is obtained from $\mathcal{Z}_{t}(s)$ of Eq. (16) (we fix $s$ to be constant for simplicity) by a Legendre transform. In a phase with a broken $\mathbb{Z}_{2}$ symmetry one might expect that the probability distribution for an appropriately chosen operator will be bimodal, analogous to a free-energy landscape with a double-well structure or the effective potential in a field theory [39]. As we show later, the operator

$$
G_{z}=\sum_{i \neq j} \sigma_{i}^{z} \sigma_{j}^{z}
$$

is such an appropriate operator.

A practical issue is that the probability distribution obtained after a naive Legendre transform cannot be multimodal as the transform preserves convexity. In addition, we see that the potential $\Theta$ constructed for the operator $G_{z}$ appears to be superextensive in the system size $L$. We now show that these two issues are both resolved by an appropriate splitting of $\mathcal{Z}$ inspired by the analysis of Ref. [40] for calculating nonconcave entropies in classical systems.

\section{A. A toy model as a limiting case}

To show how to resolve these two issues, we focus on the classical toy model defined by the limits $J_{x}=h_{\ell}=0=J_{z}$ and $J=1$ in Eq. (1) and the eigenstates of which are adiabatically connected to those in the spin glass phase of Eq. (1) and the 0 -SG phase of Sec. II. It turns out that a careful consideration of how the limits of $s \rightarrow 0$ and $L \rightarrow \infty$ [see, for instance, Eqs. (18) and (19)] is necessary, and the correct treatment gives a nonanalytic potential and a bimodal distribution. Our analysis follows the construction of nonconcave entropies in Ref. [40].

In the infinite bond disorder limit $\left(J_{x}=h_{\ell}=0=J_{z}\right.$ and $J=1$ ), the energy eigenstates are superpositions of pairs of product states which are $\mathbb{Z}_{2}$ flipped partners (in the $\sigma^{z}$ basis) of each other. In this limit, $G_{z}$ commutes with the Hamiltonian so that the energy eigenstates are also eigenstates of $G_{z}$. Labeling the eigenstates with $\varepsilon$ and \pm analogously to Sec. II A and additionally by the difference between the number of up and down spins in the state $d$, the eigenvalues of $G_{z}$ depends only upon $d$ :

$$
G_{z}|\varepsilon, \pm, d\rangle=\overline{G_{z}(d)}|\varepsilon, \pm, d\rangle
$$


where $\overline{G_{z}(d)}=\left(d^{2}-L\right) / 2$, and there are $N_{d}=\left(\begin{array}{c}L \\ (L-d) / 2\end{array}\right)$ such states in the Hilbert space. The moment-generating function for a finite system size $L$ is then

$$
\mathcal{Z}=\frac{1}{2^{L}} \sum_{d} N_{d} e^{-s t \overline{G_{z}(d)}} .
$$

Calculating $\mathcal{Z}$ and $\Theta$ for a finite system reveals a problem: one can show that in this infinite bond disorder limit

$$
\begin{gathered}
\left.\partial_{s} \Theta_{G_{z}}\right|_{s=0}=0, \\
\left.\partial_{s}^{2} \Theta_{G_{z}}\right|_{s=0}=t^{2}\left(L^{2}-L\right) / 2 \approx t^{2} L^{2} / 2,
\end{gathered}
$$

and hence the leading order, in $s$, term in $\Theta_{G_{z}} \approx s^{2} t^{2} L^{2} / 2$ is superextensive. This is unphysical as $\Theta$ is purportedly analogous to a thermodynamic potential for the out-of-equilibrium system and hence should be extensive. The issue is resolved by the same procedure that allows for $\phi$ [see Eq. (24)] to be nonconvex. We now outline this procedure.

In our calculation the thermodynamic limit $L \rightarrow \infty$ must be taken. Since $\overline{G_{z}(d)}$ may take both positive and negative values, $-L / 2 \leqslant \overline{G_{z}(d)} \leqslant L(L-1) / 2$, we split up $\mathcal{Z}$ :

$$
\begin{aligned}
\mathcal{Z} & =\mathcal{Z}^{+}+\mathcal{Z}^{-} \\
& =\frac{1}{2^{L}}\left[\sum_{|d| \leqslant\lfloor\sqrt{L}\rfloor} N_{d} e^{-s t \overline{G_{z}(d)}}+\sum_{\lfloor\sqrt{L}\rfloor<|d|<L} N_{d} e^{-s t \overline{G_{z}(d)}}\right],
\end{aligned}
$$

where the first term consists of the eigenstates for which $\overline{G_{z}(d)}>0$ while the second $\overline{G_{z}(d)}<0$. The limit $L \rightarrow \infty$ now picks out one of the two terms depending on the sign of $s$, with $\operatorname{sgn}(s)= \pm 1$ picking out $\mathcal{Z}^{ \pm}$, respectively. That is, the generating function $\mathcal{Z}$ is nonanalytic in the thermodynamic limit, with

$$
\Theta_{G_{z}}(s, t) \sim \begin{cases}\Theta_{G_{z}}^{+}(s, t)=\log \mathcal{Z}^{+}, & s>0, \\ \Theta_{G_{z}}^{-}(s, t)=\log \mathcal{Z}^{-}, & s<0 .\end{cases}
$$

The potential is to be Legendre transformed as described in Ref. [40]: If $\Phi^{ \pm}(m)$ is the Legendre transform of $\Theta^{ \pm}(s)$ [as in Eq. (24)], then the correct $\Phi(m)=\max \left[\Phi^{+}(m), \Phi^{-}(m)\right]$ might be nonconcave, as is the case for a bimodal probability distribution. It is also easy to show that $\Theta^{ \pm} \sim \pm|s| t L$ and hence the potential is extensive.

Thus, in practice, splitting the $\mathcal{Z}$ as in Eq. (32) and taking the thermodynamic limit

(i) leads to a nonanalytic $\Theta$, which in turns leads to a bimodal probability distribution,

(ii) leads to extensive potentials, and hence resolves the two issues raised at the beginning of this section. We now apply this to our quantum model.

\section{B. Numerical results for the Ising spin glass}

We numerically compute the potentials with the operator (26) for the Ising spin glass and show that the physics discussed in the previous subsection holds away from the toy limit of infinite bond disorder as well.
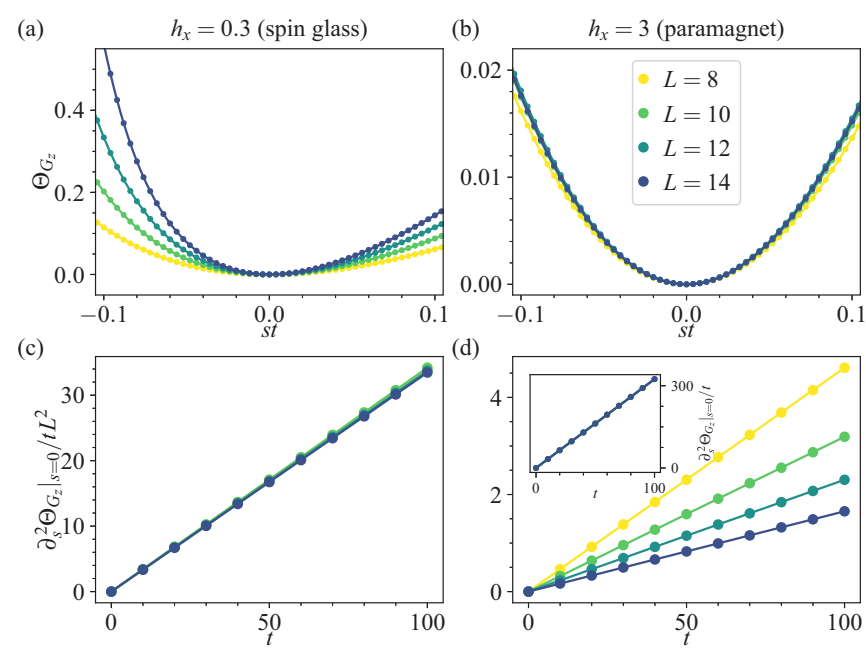

FIG. 3. The potentials $\Theta_{G_{z}}$ as a function of $s$ and $t$ in the spin glass phase (a) and the paramagnet phase (b) for various $L$. The behavior of the corresponding $\left.\partial_{s}^{2} \Theta_{G_{z}}\right|_{s=0} ^{\prime} t L^{2}$ suggests the form $\Theta_{G_{z}} \sim s^{2} t^{2} L^{2}$ in the spin glass phase (c) and $\Theta_{G_{z}} \sim s^{2} t^{2} L^{0}$ in the paramagnet phase (d) (see inset for collapse). Other parameters are $J_{z}=0, J=5, J_{x}=0.3$, and $h=0.3$.

The results for the potential $\Theta_{G_{z}}$ in the spin glass and the paramagnet phase are shown in Figs. 3(a) and 3(b), respectively. While the curvature of $\Theta_{G_{z}}$ at $s=0$ seems to grow with increasing $L$ in the spin glass phase, it does not seem to depend on the system size in the paramagnet phase. To clarify this, we explicitly look at the behavior of $\left.\partial_{s}^{2} \Theta_{G_{z}}\right|_{s=0}$ in Figs. 3(c) and 3(d).

Remarkably, in the spin glass the collapse of $\left.\partial_{s}^{2} \Theta_{G_{z}}\right|_{s=0} / L^{2}$ for various system sizes suggests that the leading contribution to $\Theta_{G_{z}}$ is of the form $\sim s^{2} t^{2} L^{2}$. As discussed in Sec. VA, this might seem alarming as $\mathcal{Z}$ plays a role analogous to the partition function and $\Theta$, the total free energy, which in this case seems to be superextensive in $L$.

The way to resolve the apparently nonphysical result that $\Theta_{G_{z}} \sim s^{2} t^{2} L^{2}$ for the full spin glass problem is then that, as discussed in Sec. VA, the limit of $L \rightarrow \infty$ should precede $s \rightarrow 0$, as is common in the treatment of problems with spontaneously broken symmetry. As it is impossible to take the limits in that order in the numerical treatment of a finite system, we separate the sum defining $\mathcal{Z}$ into branches by analogy to the example in Sec. V A, as follows.

Consider $\{|\alpha\rangle\}$ to be complete set of basis states such that $\mathcal{Z}$ can be expressed as

$$
\mathcal{Z}=\sum_{\alpha, \beta, \gamma}\left\langle\alpha\left|e^{-i \mathcal{H}^{(s)} t}\right| \beta\right\rangle\langle\beta|\rho| \gamma\rangle\left\langle\gamma\left|e^{i \mathcal{H}^{(s) \dagger} t}\right| \alpha\right\rangle .
$$

The branches are then defined by restricting the summation in Eq. (32) such that $\left\langle\alpha\left|G_{z}\right| \beta\right\rangle-\left\langle\gamma\left|G_{z}\right| \alpha\right\rangle \gtrless 0$. The results for the branches computed numerically are shown in Figs. 4(a) and 4(b). The behavior of the first derivative of $\Theta^{ \pm}$with respect to $s$ at $s=0$ in the spin glass phase shown in Fig. 4(c) suggests a leading behavior of the form $\Theta_{G_{z}}^{ \pm} \sim \pm s t L$ which is now perfectly consistent with the effective free energy being extensive in $L$. 

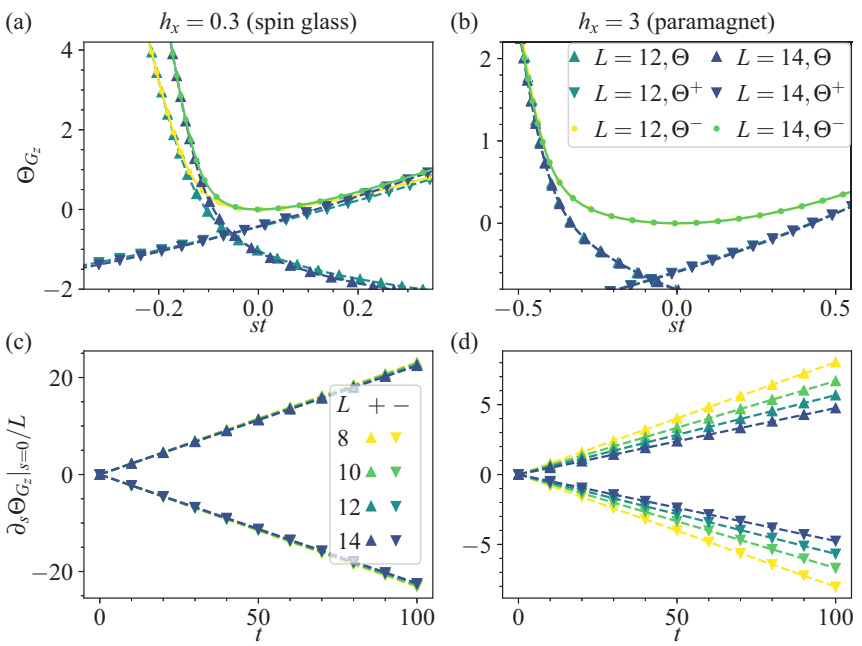

FIG. 4. The branches $\Theta_{G_{z}}^{ \pm}$for the spin glass and the paramagnet phase are superposed on $\Theta$ for two different system sizes for the spin glass phase (a) and the paramagnet phase (b). The first derivative of the branches with respect to $s$ at $s=0$ as a function of $t$ and various system sizes suggests that their leading behavior is of the form $\Theta_{G_{z}}^{ \pm} \sim$ $\pm s t L$ in the spin glass phase (c) and $\Theta_{G_{z}}^{ \pm} \sim s t L^{-1}$ in the paramagnet phase (d).

Thus the correct form of the potential $\Theta_{G_{z}}$ as the thermodynamic limit is approached is nonanalytic in the vicinity of $s=0$, yet analytic branches can be constructed, each of which is extensive in $L$. In the paramagnet phase, since the expectation of $G_{z}$ vanishes in the thermodynamic limit, the derivatives of the potential with respect to $s$ decay with increasing system size and hence the construction of branches is not necessary.

Analogous to the construction of nonconcave entropies [40], a nonanalytic $\Theta$ would suggest the presence of a nonconcave and hence multimodal distribution $P$. The probability distributions are calculated from the branches individually as $P^{ \pm} \sim e^{-L \phi^{ \pm}}$, with $\phi^{ \pm}(m, t)=-\max _{s}[s m-$ $\left.\theta^{ \pm}(s, t)\right]$, and the overall probability distribution is

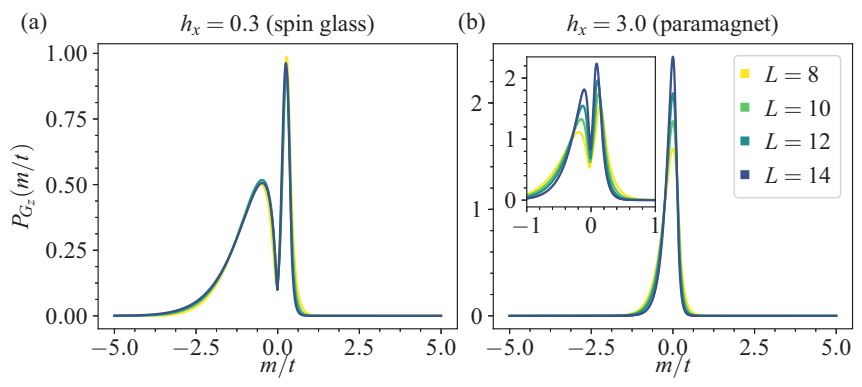

FIG. 5. The distribution $P_{G_{z}}$ showing a bimodal nature in the spin glass phase (a), which seems persistent with increasing $L$, and a unimodal distribution in the paramagnet phase (b). The inset shows that, if the branch construction is nevertheless used in the paramagnetic phase, one again obtains a bimodal distribution, but crucially the bimodality systematically goes away with increasing $L$ eventually converging to the unimodal distribution in the thermodynamic limit. reconstructed as $P(m, t)=\max \left[P^{+}(m, t), P^{-}(m)\right] / \mathcal{N}$, where $\mathcal{N}$ is the overall normalization factor. The distributions so obtained are shown in Fig. 5. In the spin glass phase the distribution is bimodal, and crucially, there is no indication of the bimodality systematically going away with increasing system size. We therefore conclude that the distribution remains bimodal in the thermodynamic limit. In the paramagnet phase, the branch construction is not used and the distribution is a unimodal one. Importantly, if one still does the branch construction in the ferromagnetic phase, a bimodal distribution is obtained for a finite system, but crucially the bimodality systematically goes away with increasing $L$ [see the inset of Fig. 5(b)]. This establishes that in the thermodynamic limit the true distribution is unimodal.

In conclusion, dynamical potentials for appropriate operators containing information about their temporal correlations show qualitatively different behaviors in systems with and without eigenstate order to the extent that they have different modalities, even at infinite temperature.

We note in closing that the operator $G_{z}$ can also be used similarly for the $\pi$ spin glass except it would show the bimodal distribution when probed at a frequency of $\omega=\pi$.

\section{CONCLUSION}

In conclusion, we have shown that appropriate spatiotemporal correlations can reflect eigenstate order and symmetry breaking at arbitrary energy densities in infinite temperature ensembles. A probe which is completely robust to initial conditions is particularly advantageous in systems open to the environment which drives the system to a mixed state involving many eigenstates, washing out eigenstate order [41-44]. It could also provide a way to get around the issue of eigenstate order getting washed out due to heating in experimental quantum simulators like cold-atom or ion-trap systems.

We then generalize the framework of Ref. [28] to mixed states and show that the dynamical potentials in infinite temperature ensembles show qualitatively different behavior in different eigenstate phases. In fact, for appropriate observables, they can also show a bimodal nature in one phase and a unimodal nature in another.

The numerical treatment presented here is restricted to finite system sizes, thus preventing us from exploring the critical points with enough accuracy. The next step in this direction is developing approximate analytical techniques, allowing access to eigenstate criticality. A natural path towards this could be recasting the moment-generating functional so as to be put on the Keldysh contour. Another direction is extending this approach to open systems, in which the time evolution of interest is itself nonunitary.

\section{ACKNOWLEDGMENTS}

We would like to thank R. Moessner and M. Heyl for useful discussions and collaboration on earlier, related work. This work is in part supported by EPSRC Grant No. EP/N01930X/1. 
[1] L. D. Landau and E. M. Lifshitz, Statistical Physics, 3rd ed., Course on Theoretical Physics Vol. 5 (Butterworth-Heinemann, Oxford, 1980).

[2] I. V. Gornyi, A. D. Mirlin, and D. G. Polyakov, Interacting Electrons in Disordered Wires: Anderson Localization and Low$T$ Transport, Phys. Rev. Lett. 95, 206603 (2005).

[3] D. M. Basko, I. L. Aleiner, and B. L. Altshuler, Metal-insulator transition in a weakly interacting many-electron system with localized single-particle states, Ann. Phys. 321, 1126 (2006).

[4] V. Oganesyan and D. A. Huse, Localization of interacting fermions at high temperature, Phys. Rev. B 75, 155111 (2007).

[5] M. Žnidarič, T. Prosen, and P. Prelovšek, Many-body localization in the Heisenberg $X X Z$ magnet in a random field, Phys. Rev. B 77, 064426 (2008).

[6] A. Pal and D. A. Huse, Many-body localization phase transition, Phys. Rev. B 82, 174411 (2010).

[7] R. Vosk, D. A. Huse, and E. Altman, Theory of the Many-Body Localization Transition in One-Dimensional Systems, Phys. Rev. X 5, 031032 (2015).

[8] D. J. Luitz, N. Laflorencie, and F. Alet, Many-body localization edge in the random-field Heisenberg chain, Phys. Rev. B 91, 081103 (2015).

[9] R. Nandkishore and D. A. Huse, Many-body localization and thermalization in quantum statistical mechanics, Annu. Rev. Condens. Matter Phys. 6, 15 (2015).

[10] Y. Bar Lev, G. Cohen, and D. R. Reichman, Absence of Diffusion in an Interacting System of Spinless Fermions on a One-Dimensional Disordered Lattice, Phys. Rev. Lett. 114, 100601 (2015).

[11] D. A. Abanin and Z. Papić, Recent progress in many-body localization, Ann. Phys. 529, 1700169 (2017).

[12] M. Schreiber, S. S. Hodgman, P. Bordia, H. P. Lüschen, M. H. Fischer, R. Vosk, E. Altman, U. Schneider, and I. Bloch, Observation of many-body localization of interacting fermions in a quasirandom optical lattice, Science 349, 842 (2015).

[13] J.-Y. Choi, S. Hild, J. Zeiher, P. Schauß, A. Rubio-Abadal, T. Yefsah, V. Khemani, D. A. Huse, I. Bloch, and C. Gross, Exploring the many-body localization transition in two dimensions, Science 352, 1547 (2016).

[14] D. A. Huse, R. Nandkishore, V. Oganesyan, A. Pal, and S. L. Sondhi, Localization-protected quantum order, Phys. Rev. B 88, 014206 (2013).

[15] D. Pekker, G. Refael, E. Altman, E. Demler, and V. Oganesyan, Hilbert-Glass Transition: New Universality of TemperatureTuned Many-Body Dynamical Quantum Criticality, Phys. Rev. X 4, 011052 (2014).

[16] J. A. Kjäll, J. H. Bardarson, and F. Pollmann, Many-Body Localization in a Disordered Quantum Ising Chain, Phys. Rev. Lett. 113, 107204 (2014).

[17] S. A. Parameswaran and R. Vasseur, Many-body localization, symmetry, and topology, Rep. Prog. Phys. 81, 082501 (2018).

[18] V. Khemani, A. Lazarides, R. Moessner, and S. L. Sondhi, Phase Structure of Driven Quantum Systems, Phys. Rev. Lett. 116, 250401 (2016).

[19] C. W. von Keyserlingk, V. Khemani, and S. L. Sondhi, Absolute stability and spatiotemporal long-range order in Floquet systems, Phys. Rev. B 94, 085112 (2016).

[20] R. Moessner and S. L. Sondhi, Equilibration and order in quantum Floquet matter, Nat. Phys. 13, 424 (2017).
[21] J. Zhang, P. W. Hess, A. Kyprianidis, P. Becker, A. Lee, J. Smith, G. Pagano, I.-D. Potirniche, A. C. Potter, A. Vishwanath et al., Observation of a discrete time crystal, Nature (London) 543, 217 (2017).

[22] S. Choi, J. Choi, R. Landig, G. Kucsko, H. Zhou, J. Isoya, F. Jelezko, S. Onoda, H. Sumiya, V. Khemani et al., Observation of discrete time-crystalline order in a disordered dipolar manybody system, Nature (London) 543, 221 (2017).

[23] S. Pal, N. Nishad, T. S. Mahesh, and G. J. Sreejith, Temporal Order in Periodically Driven Spins in Star-Shaped Clusters, Phys. Rev. Lett. 120, 180602 (2018).

[24] J. Rovny, R. L. Blum, and S. E. Barrett, Observation of DiscreteTime-Crystal Signatures in an Ordered Dipolar Many-Body System, Phys. Rev. Lett. 120, 180603 (2018).

[25] H. Haken and W. Weidlich, A theorem on the calculation of multi-time-correlation functions by the single-time density matrix, Z. Phys. 205, 96 (1967).

[26] L. O. Hedges, R. L. Jack, J. P. Garrahan, and D. Chandler, Dynamic order-disorder in atomistic models of structural glass formers, Science 323, 1309 (2009).

[27] J. M. Hickey, S. Genway, I. Lesanovsky, and J. P. Garrahan, Time-integrated observables as order parameters for full counting statistics transitions in closed quantum systems, Phys. Rev. B 87, 184303 (2013).

[28] S. Roy, A. Lazarides, M. Heyl, and R. Moessner, Dynamical potentials for nonequilibrium quantum many-body phases, Phys. Rev. B 97, 205143 (2018).

[29] T. A. Kaplan, P. Horsch, and W. Von der Linden, Order parameter in quantum antiferromagnets, J. Phys. Soc. Jpn. 58, 3894 (1989).

[30] T. Koma and H. Tasaki, Symmetry breaking in Heisenberg antiferromagnets, Commun. Math. Phys. 158, 191 (1993).

[31] L. D'Alessio and M. Rigol, Long-Time Behavior of Isolated Periodically Driven Interacting Lattice Systems, Phys. Rev. X 4, 041048 (2014).

[32] A. Lazarides, A. Das, and R. Moessner, Equilibrium states of generic quantum systems subject to periodic driving, Phys. Rev. E 90, 012110 (2014).

[33] P. Ponte, A. Chandran, Z. Papić, and D. A. Abanin, Periodically driven ergodic and many-body localized quantum systems, Ann. Phys. 353, 196 (2015).

[34] A. Lazarides, A. Das, and R. Moessner, Fate of Many-Body Localization under Periodic Driving, Phys. Rev. Lett. 115, 030402 (2015).

[35] P. Ponte, Z. Papić, F. Huveneers, and D. A. Abanin, Many-Body Localization in Periodically Driven Systems, Phys. Rev. Lett. 114, 140401 (2015).

[36] P. Bordia, H. Lüschen, U. Schneider, M. Knap, and I. Bloch, Periodically driving a many-body localized quantum system, Nat. Phys. 13, 460 (2017).

[37] M. Reitter, J. Näger, K. Wintersperger, C. Sträter, I. Bloch, A. Eckardt, and U. Schneider, Interaction Dependent Heating and Atom Loss in a Periodically Driven Optical Lattice, Phys. Rev. Lett. 119, 200402 (2017).

[38] A. Eckardt, Colloquium: Atomic quantum gases in periodically driven optical lattices, Rev. Mod. Phys. 89, 011004 (2017).

[39] G. Mussardo, Statistical Field Theory: An Introduction to Exactly Solved Models in Statistical Physics (Oxford University, London, 2010).

[40] H. Touchette, Methods for calculating nonconcave entropies, J. Stat. Mech.: Theory Exp. (2010) P05008. 
[41] M. V. Medvedyeva, T. Prosen, and M. Žnidarič, Influence of dephasing on many-body localization, Phys. Rev. B 93, 094205 (2016).

[42] M. H. Fischer, M. Maksymenko, and E. Altman, Dynamics of a Many-Body-Localized System Coupled to a Bath, Phys. Rev. Lett. 116, 160401 (2016).
[43] E. Levi, M. Heyl, I. Lesanovsky, and J. P. Garrahan, Robustness of Many-Body Localization in the Presence of Dissipation, Phys. Rev. Lett. 116, 237203 (2016).

[44] A. Lazarides and R. Moessner, Fate of a discrete time crystal in an open system, Phys. Rev. B 95, 195135 (2017). 